\title{
New Catalysis Concepts for Molecular Design and Feedstocks Valorization
}

\author{
Philip Boehm and Bill Morandi*
}

\begin{abstract}
The discovery and preparation of important molecules rely on the synthetic chemist's ability to valorize common feedstock materials, such as petroleum or biomass. Key to this endeavor is the design of efficient methods to introduce, remove or interconvert functional groups. Our group has a longstanding interest in developing conceptually novel catalytic reactions for the installation of unprotected amines, the selective activation of $\mathrm{C}-\mathrm{O}$ bonds (in polyols) and the transfer (shuttle) or exchange (metathesis) of functionalities between two molecules. This account describes representative examples from our group in these different research areas.
\end{abstract}

Keywords: Amination - C-O bond activation · Homogeneous catalysis · Isodesmic reactions · Metathesis

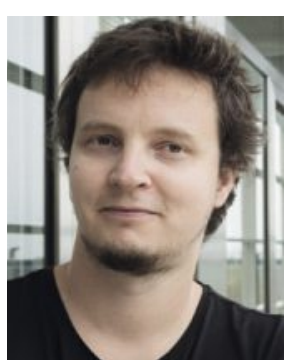

Bill Morandi was born in Fribourg, Switzerland. He studied at the ETH Zurich (2003-2008), receiving a BSc in Biology and a MSc in Chemical Biology. From 2008 until 2012, he pursued his PhD in the labs of Prof. Erick M. Carreira, working on diazo compounds. Afterwards, he moved to the California Institute of Technology for a SNSF-funded postdoctoral stay with Prof. Robert H. Grubbs. During this time, he was studying the oxidation of internal alkenes. In 2014, he became an independent Max Planck Research Group Leader at the MaxPlanck-Institut für Kohlenforschung in Mülheim/Ruhr, Germany. Since July 2018, Bill Morandi is an Associate Professor (with tenure) at the ETH Zurich where he holds a chair of synthetic organic chemistry at the Laboratorium für Organische Chemie (D-CHAB).

\section{Introduction}

The vast majority of important organic molecules rely on the presence of precisely located functional groups to control their physical properties, their interaction with their surrounding environment, and, ultimately, their function. The manipulation of functional groups - introduction, removal, interconversion - has thus been at the core of synthetic organic chemistry since its inception. ${ }^{[1]}$ For us organic chemists, there is, however, only a limited set of feedstock chemicals that are broadly available for the synthesis of high-value compounds, calling for the development of efficient methods to transform them into most-needed pharmaceuticals, agrochemicals and organic materials that benefit the life of billions of people worldwide.

Nowadays, most chemicals originate from fossil feedstocks, which mostly consist of simple hydrocarbons lacking the polar functional groups often found in useful molecules. The invention of novel methods for the efficient and selective functionalization of hydrocarbons has thus been an essential and longstanding goal of organic chemistry. More recently, the need to develop new methods to transform abundant, renewable biomass, such as carbohydrates or lignin, has driven the development of new concepts and strategies in organic synthesis. In contrast to fossil feedstocks, biomass-derived chemicals tend to be overly func- tionalized when compared to many desired synthetic intermediates or final products. As suggested by Prof. Bergman: "Whereas the preparation of useful chemicals from petroleum has historically been accomplished by adding heteroatom functionality to unfunctionalized starting materials, the conversion of biomass commonly requires the removal of oxygen atoms from the natural precursors."'[2]

Catalysis is a powerful tool that can be used for the selective (de-)functionalization of molecules, thus streamlining the synthesis of the desired compounds in a sustainable manner. Our group has been mainly engaged in the development of new catalytic methods for the manipulation of functional groups. This short account describes some of our recent efforts to functionalize hydrocarbon feedstocks through amination reactions which, in contrast to most literature precedent, do not rely on protecting group strategies. We also present new catalytic reactions for the selective, partial deoxygenation of simple polyols. Finally, we discuss the development of a host of catalytic reversible reactions that include both the transfer of a group (shuttle catalysis) or the exchange of two functional groups (metathesis), many of which allow for novel disconnections or improved safety profiles.

\section{Introducing Functional Groups - Direct Synthesis of Unprotected Primary Amines from Hydrocarbons}

Given the importance of amines in natural product synthesis and medicinal chemistry, the installation of carbon-nitrogen bonds still remains one of the major challenges in organic synthesis. The direct catalytic amination of hydrocarbons represents a powerful approach to address this challenge and has been studied extensively. ${ }^{[3]}$ However, the majority of the synthetic protocols developed thus far lead to the installation of a protected form of the versatile primary amino group, a feature complicating synthetic sequences with wasteful protecting group manipulations. Major challenges in the synthesis of unprotected primary amines are undesired side reactions or irreversible coordination of the product to the metal catalyst, leading to its deactivation. If such challenges can be addressed, the introduction of a free $\mathrm{NH}_{2}$-group would streamline the synthesis of target molecules, e.g. in medicinal chemistry. Therefore, our group, amongst others, is investigating the synthesis of unprotected amines from unactivated hydrocarbons using inexpensive iron catalysts. ${ }^{[4]}$ 
Amino alcohols play a privileged role in medicinal chemistry and their efficient synthesis is highly sought after. The aminohydroxylation of alkenes is a powerful method for the preparation of these scaffolds from simple feedstocks. ${ }^{[5]}$ However, the conventional methods are limited by the necessity to introduce a protected version of the amino group to ensure an efficient reactivity. Since these protecting groups, $e . g$. tosyl, usually need to be cleaved prior to subsequent modifications, we reasoned that a method providing direct access to both the free amino and hydroxy groups would facilitate the preparation of medicinally relevant nitrogen compounds. Our group has thus developed a mild iron-catalyzed synthesis of unprotected amino alcohols from alkenes using an easily accessible hydroxylamine-derived reagent and water (Scheme 1a). ${ }^{[6]}$ Amongst the catalysts tested, inexpensive $\mathrm{Fe}^{\mathrm{II}}$ phthalocyanine proved to be the best to provide the aminohydroxylated products in good yields with complete regioselectivity. The transformation is particularly suited for the preparation of 2-amino-1-phenylethanols, a structural motif present in over 20 approved drugs.

Recently, we have been able to develop a related iron-based catalytic system for the aminochlorination of a wide range of alkenes (Scheme 1b). ${ }^{[7]}$ Both activated and unactivated alkenes could be transformed into the corresponding 2-chloroamine products with the same hydroxylamine reagent mentioned above and sodium chloride in the presence of an $\mathrm{Fe}^{\mathrm{II}}$ catalyst. The products show amphoteric character and can be further transformed into a great variety of nitrogen-containing products. Importantly, this new method tolerates a vast scope of unactivated alkenes including several drug-like moieties.

Besides aminoalcohols, 2-azidoamines are another attractive target in the difunctionalization of alkenes, as they are important precursors to orthogonally-substituted vicinal diamines. We have recently developed an iron-catalyzed aminoazidation of a broad range of alkenes, including complex drug-like molecules. ${ }^{[8]}$ The synthetic potential of this reaction could be shown in the orthogonal derivatization of the unprotected azidoamine group to access a plethora of vicinal diamine derivatives (Scheme 1c).

The recent discovery of a new reaction for the amination of aromatic substrates further expands the repertoire of nitrogencontaining compounds that are accessible through our methods. Simple $\mathrm{Fe}^{\mathrm{II}}$ sulfate and a slightly modified hydroxylamine reagent enable the direct, innate amination of arenes to access synthetically important primary anilines (Scheme 1d). ${ }^{[9]}$ This transformation can facilitate the amination of a wide range of substrates, including complex bioactive scaffolds. More importantly, this work is a rare example of a $\mathrm{C}-\mathrm{H}$ amination reaction leading directly to the formation of unprotected primary anilines. ${ }^{[10]}$ Further optimization of these reaction conditions by the Ritter group has led to an expanded substrate scope including electron-deficient substrates using HFIP as a solvent. ${ }^{[10 a]}$

The reactions developed during our research program give access to a variety of unprotected amines from simple hydrocarbon starting materials. By using inexpensive iron catalysts and hydroxylamine derived reagents, the aminohydroxylation, aminochlorination and aminoazidation of alkenes, as well as the $\mathrm{C}-\mathrm{H}$ amination of arenes, have been achieved. Very recently, we introduced a new family of substituted hydroxylamine-derived reagents for the direct synthesis of unprotected secondary amines as well as tertiary amines using a similar approach, thus considerably increasing the synthetic potential of these reactions. ${ }^{[11]}$ In all cases mentioned above, the unprotected amine is obtained as the product, making protecting group manipulations unnecessary and thus streamlining the synthesis of important compounds.

\section{Removing Functional Groups - Selective Activation of Aliphatic C-O Bonds}

The alcohol group is one of the most common functional groups in organic synthesis. Additionally, hydroxy groups are

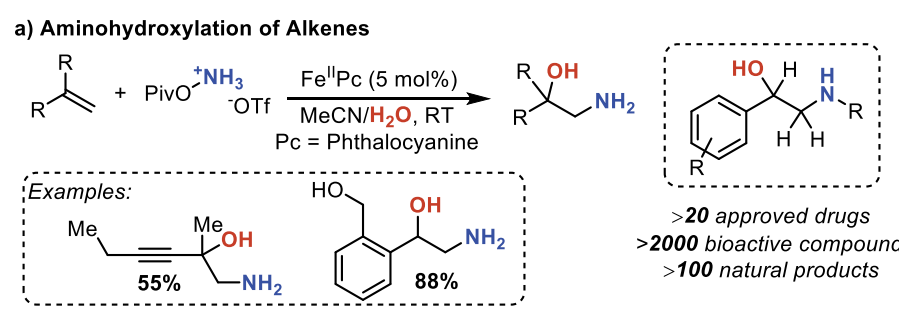

b) Aminochlorination of Alkenes
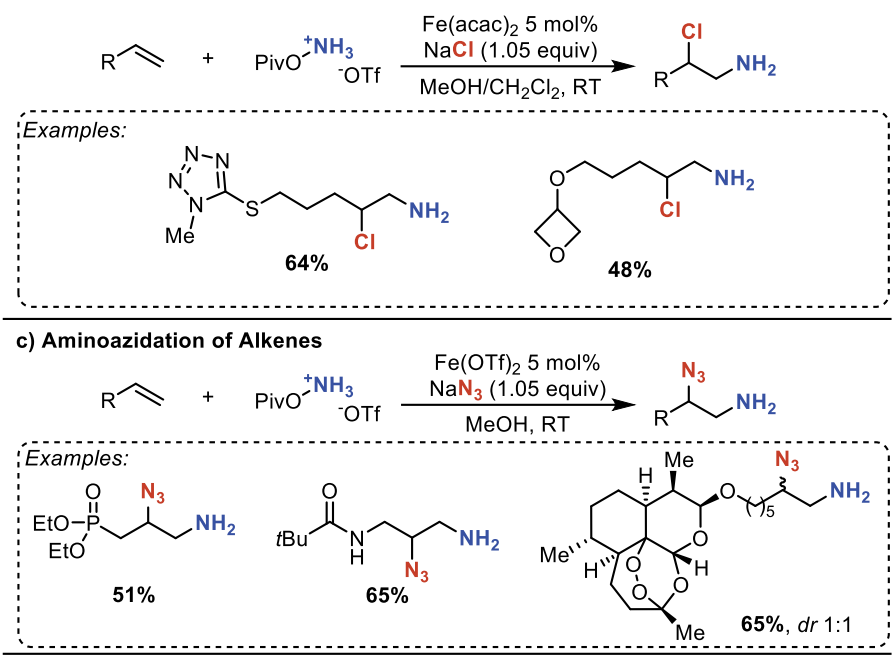

d) C-H Amination of Arenes
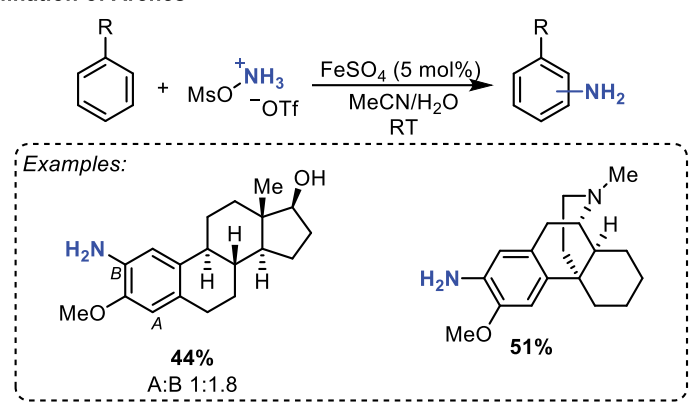

Scheme 1. Methods for the synthesis of unprotected amines.

ubiquitous in renewable feedstocks, ${ }^{[12]}$ such as carbohydrate derivatives. However, these renewable feedstocks tend to contain more hydroxyl functionalities than usually needed for downstream synthetic applications. Therefore, methods for the transformation and functionalization of alcohols are extremely useful in synthesis. The site-selective deoxygenation of a specific hydroxy group in polyols remains an important challenge in organic synthesis. ${ }^{[13]}$ Our group is thus interested to develop novel transformations employing alcohol derivatives as starting materials, with a particular focus on selectively manipulating polyols.

Initially, we explored the regioselective deoxygenation of terminal diols, as this group is often present in renewable feedstocks and synthetic intermediates. We have developed a selective monodeoxygenation of 1,2-terminal diols at the primary position using a simple, commercially available boron catalyst and hydrosilane reagents. ${ }^{[14]}$ As a core design, the formation of a cyclic intermediate which enhances the rate of the first deoxygenation step helps prevent over-reduction to the alkane. Using this strategy, we could deoxygenate several diols and even access a highly enantioenriched 2-alkanol product that was further used in the enantioselective synthesis of $(R)$-lysofylline, an anti-inflammatory compound (Scheme 2a). Computational studies performed in collaboration with the Thiel group have revealed that the cyclic siloxane intermediate promotes the first deoxygenation step as it experiences less distortion in the transition state and leaves more space for the attacking hydride. The first deoxygenation step generates a stable 
product due to the release of ring strain and creates a large steric bulk on the secondary alcohol that prevents over-reduction. ${ }^{[15]}$

Using a similar catalyst system as in the selective deoxygenation of terminal diols, a rare example of reductive pinacol-type rearrangement of unactivated internal diols could also be achieved (Scheme 2b). ${ }^{[16]}$ The reaction provides novel synthetic avenues for the construction of $\alpha$-substituted alcohols. A broad scope of scaffolds is tolerated, including cyclic diols that can undergo either ring expansion or contraction. As shown in mechanistic experiments, the reaction proceeds via a concerted 1,2-migration with inversion of the stereochemistry. To explain the difference in chemoselectivity between deoxygenation of terminal diols versus rearrangement with internal diols, computational studies were conducted. The results show that in the case of terminal diols, the deoxygenation is kinetically favored over migration. In the case of internal diols, the migration is more favorable due to the hyperconjugative and steric effects of the additional substituent.[16]

Intrigued by this enhanced reactivity of cyclic intermediates in $\mathrm{C}-\mathrm{O}$ bond reduction, we reasoned that mechanistically distinct $\mathrm{C}-\mathrm{O}$ bond cleavage reactions could also benefit from this effect for the transformation of polyols. In addition to cyclic siloxanes, cyclic sulfates are an equally easily accessible class of electrophiles derived from abundant 1,2-diols. ${ }^{[17]}$ Under copper catalysis, a Kumada-type coupling of cyclic sulfates was developed for the synthesis of a wide range of functionalized alcohol products in high regio- and chemoselectivity (Scheme 2c). ${ }^{[18]}$ It is further demonstrated that the cyclic sulfate group reacts preferentially over the chemically related tosylate group, showcasing the activating effect of the cyclic intermediate. In addition, a sugar derivative undergoes a successful site-selective coupling of a single hydroxy group.

These results in the selective carbon-oxygen bond activation illustrate the long-term goal of this project, namely the synthesis of value-added building blocks from biomass, e.g. carbohydrates or lignin.

\section{Transferring Functional Groups - Shuttle Catalysis and Metathesis}

Catalytic reversible reactions, such as alkene metathesis ${ }^{[19]}$ and transfer hydrogenation, ${ }^{[20]}$ have had a lasting impact on the molecular sciences and found numerous applications, e.g. in the field of natural product synthesis ${ }^{[21]}$ or material sciences. ${ }^{[22]}$ Inspired by those fundamental reactions, shuttle catalysis, the catalytic, reversible transfer of functional groups between two molecules, has emerged as a powerful tool for the construction and deconstruction of organic compounds. ${ }^{[23]}$ By paralleling the mechanism of transfer hydrogenation, we try to address important synthetic challenges and provide new disconnections for synthetic chemists. One important goal in this program is to achieve reversible incorporation of functional groups as a complement to the other projects performed in our research group. Additionally, we are seeking to employ similar principles to develop single-bond metathesis reactions to realize powerful applications across the molecular sciences.

We initially targeted the development of a catalytic reversible hydrofunctionalization reaction of alkenes. The hydrocyanation reaction was selected as a platform to develop this concept because alkenes and nitriles are versatile synthetic intermediates and show complementary reactivity profiles. Furthermore, the laboratoryscale hydrocyanation reaction has been underexploited because traditional approaches often rely on the use of volatile and highly toxic hydrogen cyanide $(\mathrm{HCN})$ as a reagent. ${ }^{[24]}$ Additionally, the reverse retro-hydrocyanation is not known because it is thermodynamically disfavored. To address these challenges, we have reported a transfer hydrocyanation reaction wherein alkenes and nitriles are efficiently interconverted in the presence of a Ni-catalyst and a Lewis acid (Scheme 3a). ${ }^{25]}$ This strategy eludes the need for

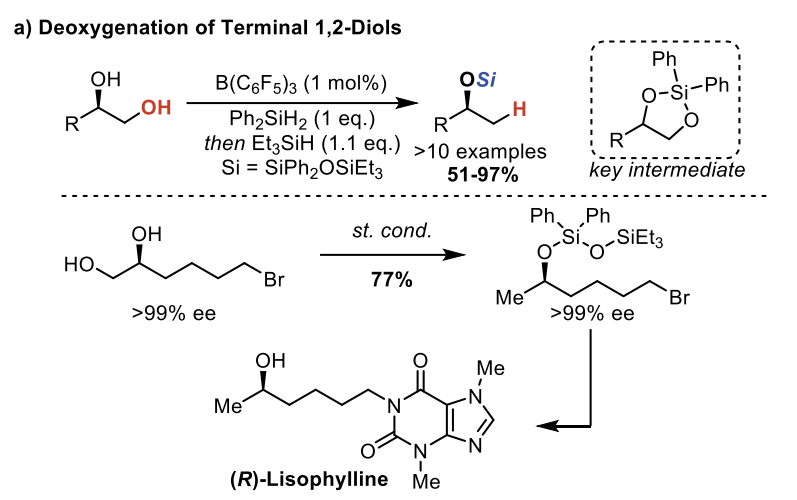

b) Reductive Pinacol-Type Rearrangement of Internal 1,2-Diols

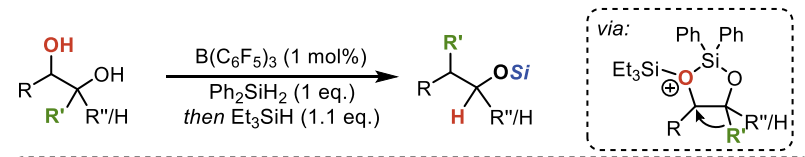

Examples:

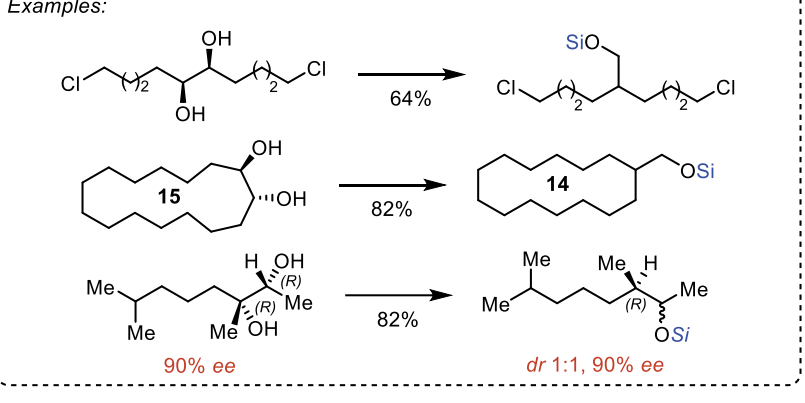

c) Kumada-Coupling of Cyclic Sulfate Esters
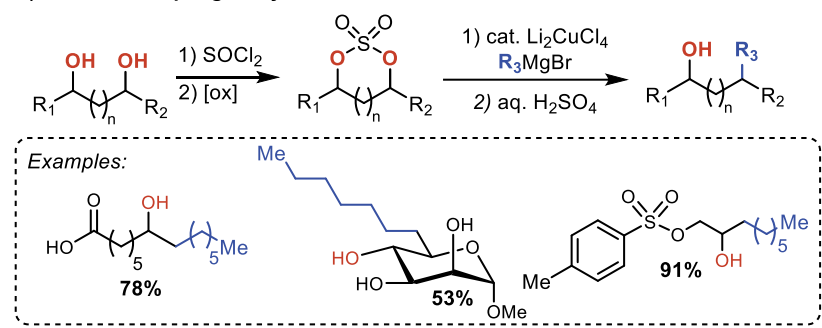

Scheme 2. Methods for selective C-O bond activation.

using hydrogen cyanide as a reagent, and thus provides a safer approach to hydrocyanation reactions when compared to traditional methods. This reaction is also a rare example of transfer functionalization which can be fully controlled using simple driving forces, such as strain release or gas extrusion, to favor, at will, either the forward or reverse reaction. More recently, we have developed a kinetically controlled, yet still reversible variant of the transfer hydrocyanation to obtain branched nitriles. ${ }^{[26]}$ We have also been interested in harnessing the reversibility of the transfer hydrocyanation to construct new catalytic cycles, for example for the cyanation of aryl halides. ${ }^{[27]} \mathrm{A}$ central transmetallation between two independently generated $\mathrm{Ni}^{\mathrm{II}}$ species, from dehydrocyanation of butyronitrile and oxidative addition into the aryl (pseudo)halide, is proposed (Scheme $3 b$ ). The reaction shows broad functional group tolerance and also overcomes common drawbacks of other cyanation reactions, such as catalyst deactivation by cyanide.

To further expand the scope of transfer hydrofunctionalization reactions, we envisioned the possibility to perform a transfer hydrochlorocarbonylation of alkenes and alkynes. Indeed, under palladium catalysis, using inexpensive butyryl chloride as a formal $\mathrm{HCl}$ and $\mathrm{CO}$ donor, a wide range of alkynes and alkenes could be transformed into the corresponding acid chloride products (Scheme 4a). ${ }^{[28]}$ The acid chlorides can then be converted in situ into a diverse array of carboxylic acid derivatives and ketones. The reaction circumvents the use of toxic, pressurized carbon mon- 


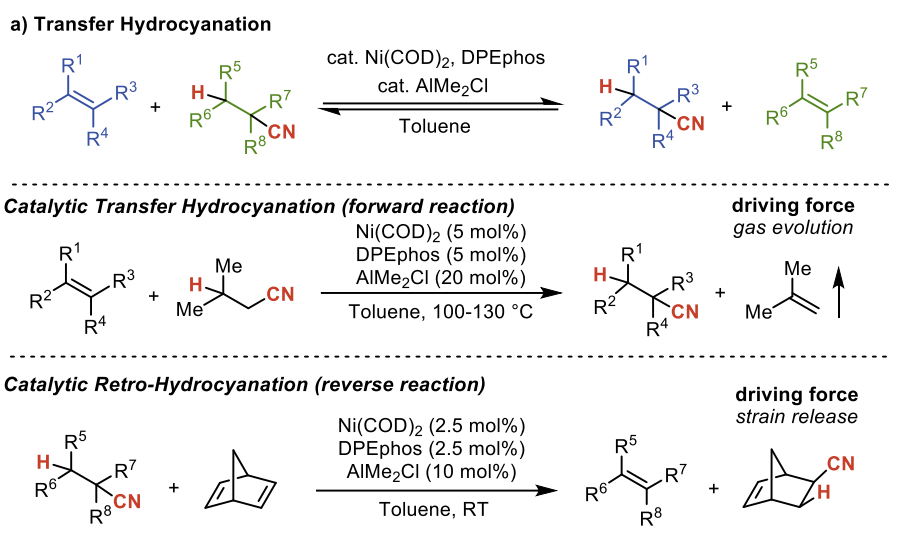

b) Cyanation of Aryl Chlorides and Triflates using Butyronitrile
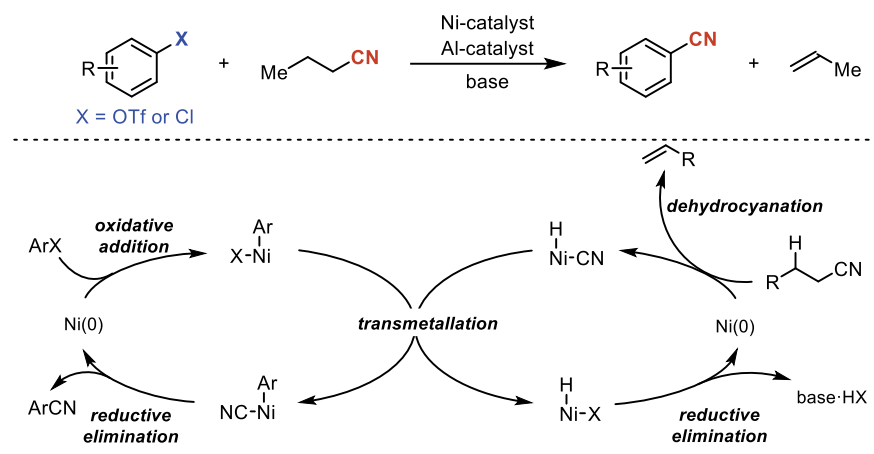

Scheme 3. Transfer hydrocyanation and related transformations.

oxide that is usually required in traditional Reppe-type carbonylation chemistry. Furthermore, only a single catalyst system is required to provide access to a wide spectrum of carbonyl containing products through the incorporation of various nucleophiles. As acid chlorides exhibit high electrophilicity, nucleophiles that were considered as unreactive due to steric encumbrance or low coordinating ability could be employed in this reaction as well.

Recently, we reported an iridium-catalyzed hydrochlorination and hydrobromination of alkynes (Scheme 4b). ${ }^{[29]}$ By using either 4-chlorobutan-2-one or tert-butyl chloride for the hydrochlorination or tert-butyl bromide for the hydrobromination, a diverse array of alkynes could be converted into the corresponding vinyl halides under iridium-catalysis. With this method, the use of corrosive hydrogen halides can be circumvented, not only improving the safety profile of this reaction, but also its functional group tolerance. For instance, acid labile groups such as acetals, tertiary alcohols or silyl ethers were tolerated under the reaction conditions.

In a recent breakthrough, we were able to overcome a major limitation of shuttle catalysis reactions by developing a transfer difunctionalization reaction in collaboration with the Waldvogel group. ${ }^{[23,30]}$ The transfer of two functional groups was realized via an electrochemical shuttle (coined $e$-shuttle) approach proceeding through paired electrolysis using inexpensive graphite electrodes. Using a simple reaction setup which is accessible to non-specialized laboratories, a broad range of alkenes can be converted into their dichlorinated or dibrominated analogs, using inexpensive 1,2-dichloroethane or 1,2-dibromoethane as $\mathrm{Cl}_{2}$ and $\mathrm{Br}_{2}$ surrogates, respectively (Scheme 4c). In a further synthetic application, it was shown that lindane, a once world-widely used insecticide, which is now classified as a persistent organic pollutant, could be efficiently degraded to benzene in three subsequent retro-dichlorination events, while simultaneously acting as a $\mathrm{Cl}_{2}$ source in a synthetically useful transfer dichlorination reaction. Lindanecontaminated soils could also be used directly, without any pretreatment, as donors in this reaction highlighting the potential of this approach for combining the recycling of wastes with the synthetically relevant preparation of halogenated molecules. ${ }^{[31]}$ a) Transfer Hydrochlorocarbonylation of Alkenes and Alkynes
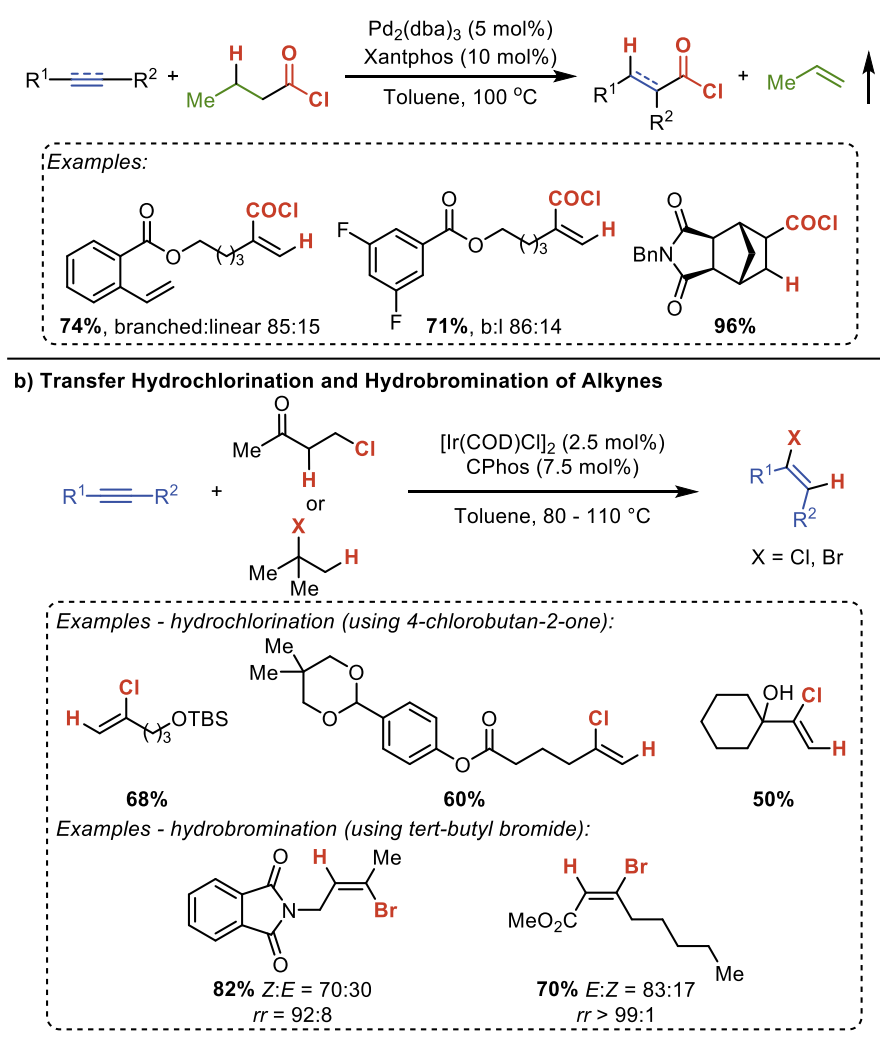

c) Electrochemical Transfer Dichlorination and Dibromination

Scheme 4. Transfer hydrochlorocarbonylation and hydrohalogenation of alkynes enabled by shuttle catalysis.

The principle of shuttle catalysis can be extended to the concomitant exchange of two different shuttled groups through a formal functional group metathesis process. Based on this concept, our group, simultaneously with the Arndtsen group, has recently realized such a transformation using a Pd catalyst to swap an acid chloride and an iodide functional group (Scheme 5). ${ }^{[32]}$ Besides its conceptual novelty, the reaction can be used as a CO-free alternative for the chlorocarbonylation of aryl iodides. Different driving forces, e.g. steric crowding or electronic destabilization, are used to shift the equilibrium of the reaction towards the desired products. Mechanistic studies suggest that this reaction is enabled by an unusual type of ligand non-innocence, wherein the phosphine ligand coordinated to the transition metal acts as a temporary aryl group storage unit through a rapid $\mathrm{C}-\mathrm{P}$ bond metathesis event proceeding at the ligand itself.

In related efforts, we have been interested in expanding the concept of single bond metathesis to other functional groups. We have developed a $\mathrm{C}-\mathrm{S} / \mathrm{H}-\mathrm{S}$ bond metathesis reaction that enables the facile modification of thioethers, an important class of compounds for medicinal and materials applications, through a palladium-catalyzed, reversible $\mathrm{C}-\mathrm{S}$ oxidative addition (Scheme 6a) ${ }^{[33]}$ In this case the two shuttled groups belong to the same class of functional groups and only differ in their substitution patterns. In addition to a broad functional group tolerance, the reaction 


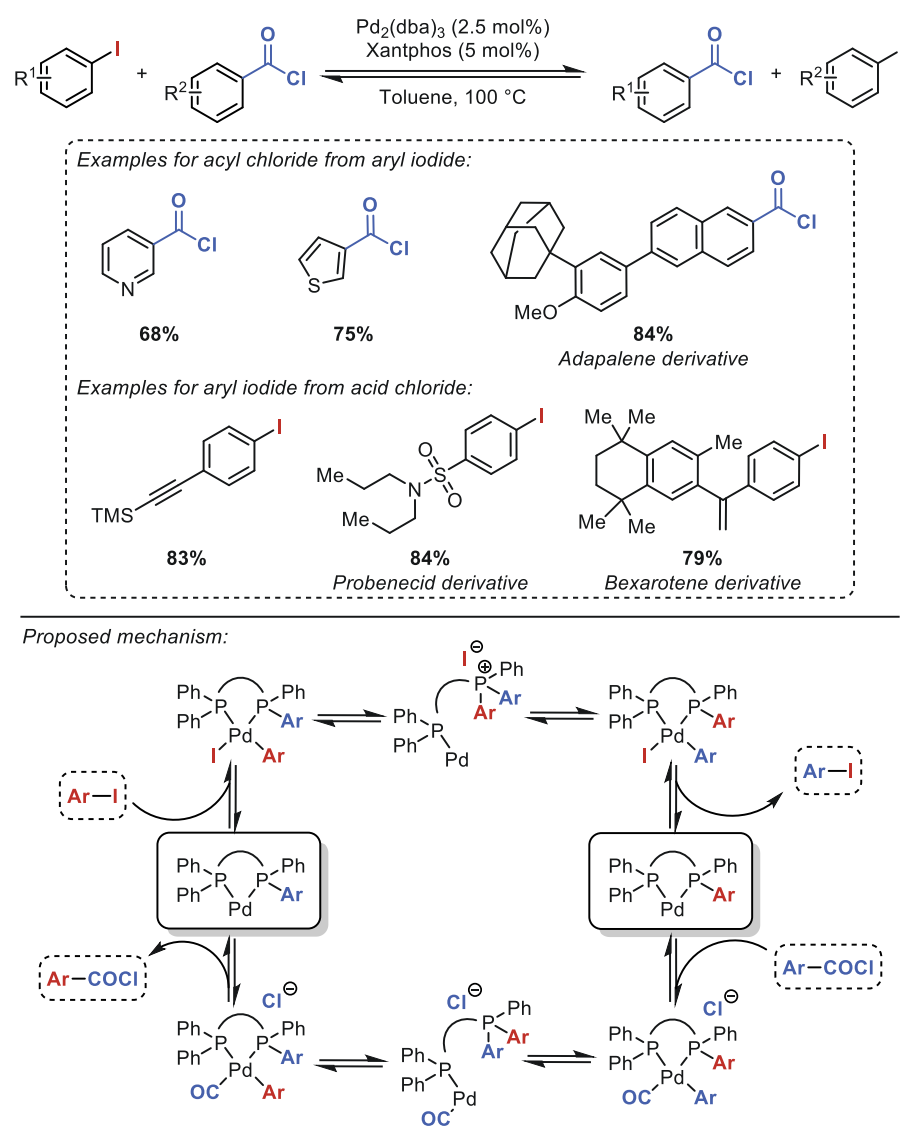

Scheme 5. Palladium-catalyzed functional group metathesis between aryl iodides and aroyl chlorides.

could be used for the late-stage diversification of a commercially available drug molecule and the depolymerization of a bulk thermoplastic polymer, demonstrating the interdisciplinary synthetic potential of this class of single bond metathesis reactions. Recently, our carbon-sulfur bond metathesis could be improved by using a more sustainable nickel catalyst (Scheme 6b) ${ }^{[34]}$ Not only could the substrate scope be broadened, but the reaction also proved to be viable in the synthesis of novel macrocyclic sulfurcontaining heterocycles. In-depth mechanistic studies suggest a $\mathrm{Ni}(0) / \mathrm{Ni}(\mathrm{II})$ catalytic cycle with the precipitation of a lithium thiolate salt as driving force for the reaction.

Excitingly, single bond metathesis is not limited to $\mathrm{C}-\mathrm{S}$ bonds. A C-P/C-P bond metathesis reaction using a catalytic amount of $\mathrm{PhI}$ as an initiator (Scheme 7a) could be realized as well. ${ }^{[33]}$ In this reaction, the phenyl iodide additive serves to generate phosphonium intermediates that can undergo facile and reversible oxidative addition, enabling the exchange of the aryl substituents among the starting materials. Besides showing the basic principle of this reaction in simple examples of cross-metathesis, we also applied it to the preparation of a new chiral ligand in a single step starting from commercially available stating materials. Furthermore, the corresponding ring closing methodology enabled us to discover a new molecule with interesting photophysical properties (strong fluorescence and solvatochromic behavior) that bodes well for its application as an organic material.

Using a simple $\mathrm{Fe}(\mathrm{III})$ catalyst, the ring-closing $\mathrm{C}-\mathrm{O} / \mathrm{C}-\mathrm{O}$ metathesis of aliphatic ethers could also be achieved in our laboratories (Scheme 7b).[35] Through this method, substituted tetrahydrofurans, tetrahydropurans, morpholines and polycyclic ethers could be accessed. Initial mechanistic studies suggest a mechanism which is initiated by coordination of the Lewis acidic iron catalyst to one of the oxygen atoms of the substrate. The non-coordinated oxygen atom can attack in an intramolecular fashion to provide an intermediate oxonium and an iron methoxide species.

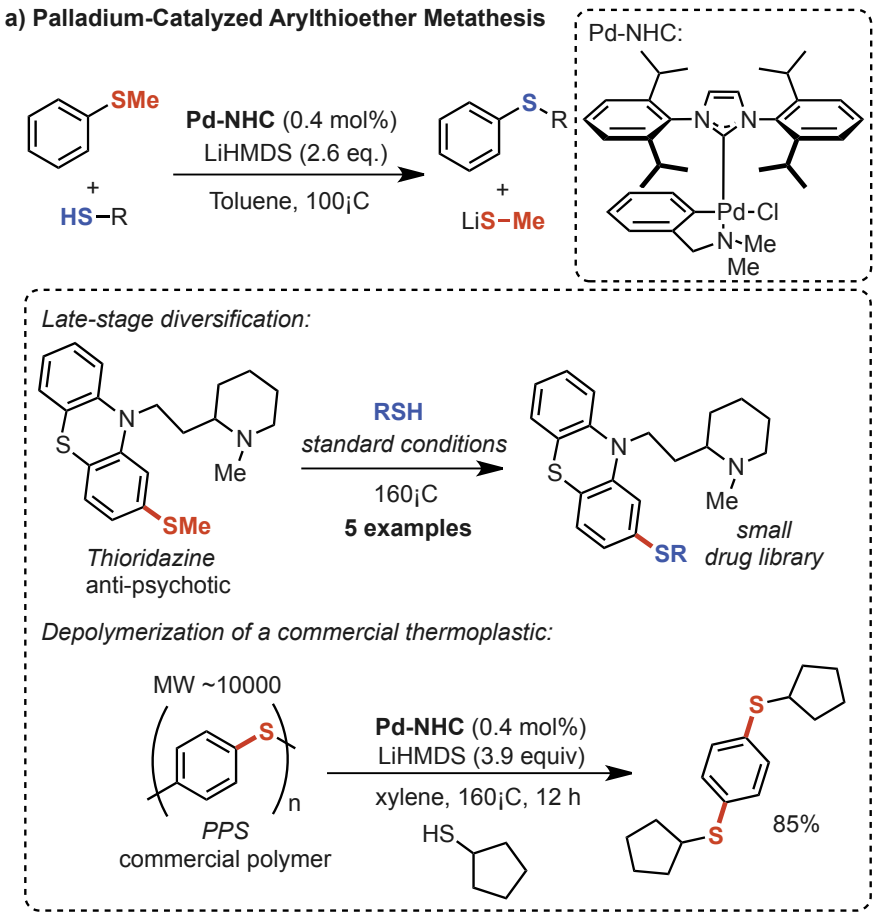

b) Nickel-Catalyzed Arylthioether Metathesis
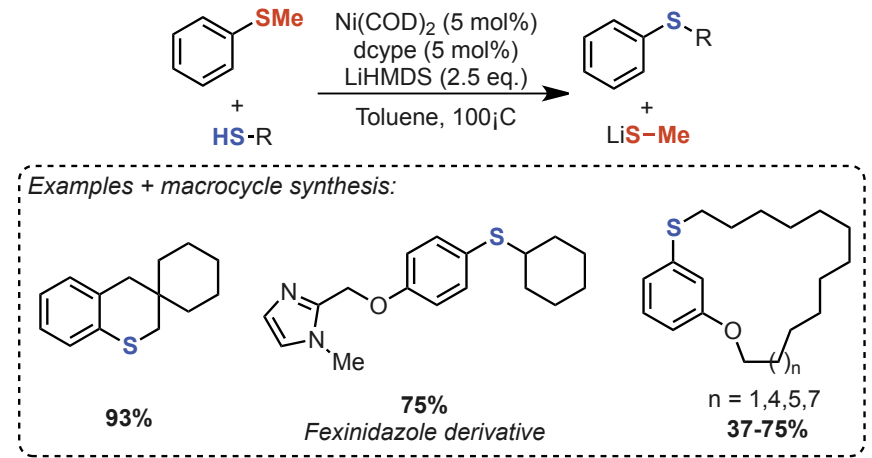

Scheme 6. Arylthioether metathesis.

The methoxide can then demethylate the oxonium ion to provide the cyclic ether product and dimethyl ether.

\section{Conclusion and Outlook}

Herein, we have illustrated our group's research interests in the manipulation of functional groups for the valorization of common feedstock chemicals. While these initial results are promising, there are many challenges that lie ahead for research in these areas.

With regards to the functionalization of hydrocarbons, we plan to expand the project to the direct primary amination of simple aliphatic alkanes. We also look forward to apply some of the reactions developed, such as the aminoazidation reaction, to more complex biomolecules given the exceptional functional group tolerance of these reactions. We also seek to gather mechanistic information about this rather complex catalytic system in collaboration with experts in EPR and Mössbauer spectroscopy.

Selective polyol (de-)functionalization will also continue to be an important topic in research, both in our group and in the community at large. We are both interested to develop new methods for selective functionalization of simple model polyols as well as trying to apply these methods to the valorization of biomassderived feedstocks, such as carbohydrates. This is a very stimulating topic as both the reactivity (how to catalytically activate $\mathrm{C}-\mathrm{O}$ bonds?) and site-selectivity challenges will require completely new approaches and concepts to be developed. 


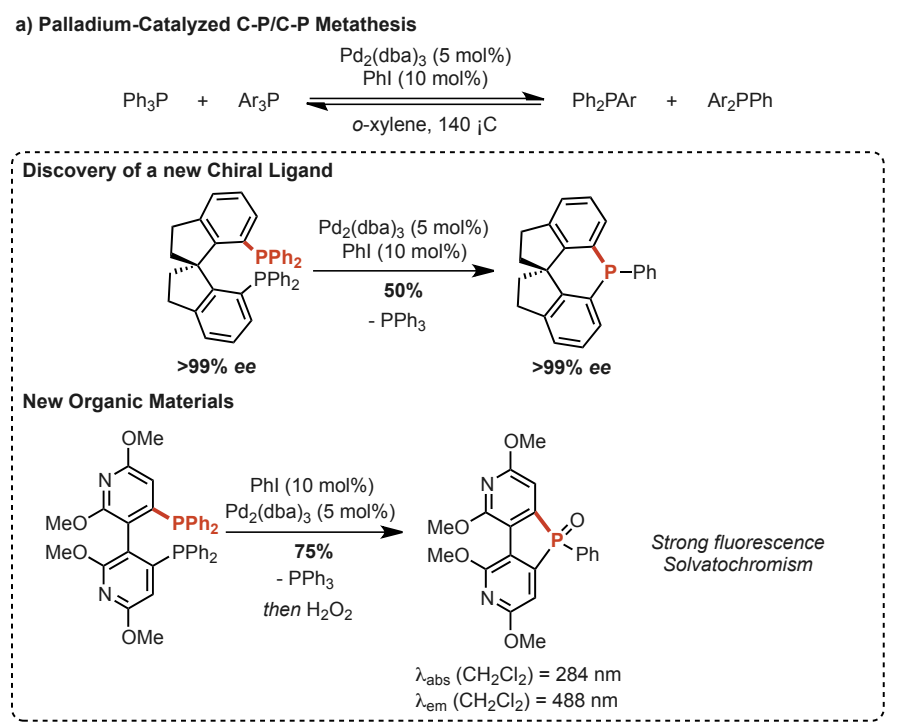

b) Iron-Catalyzed Ring-Closing C-O/C-O Metathesis

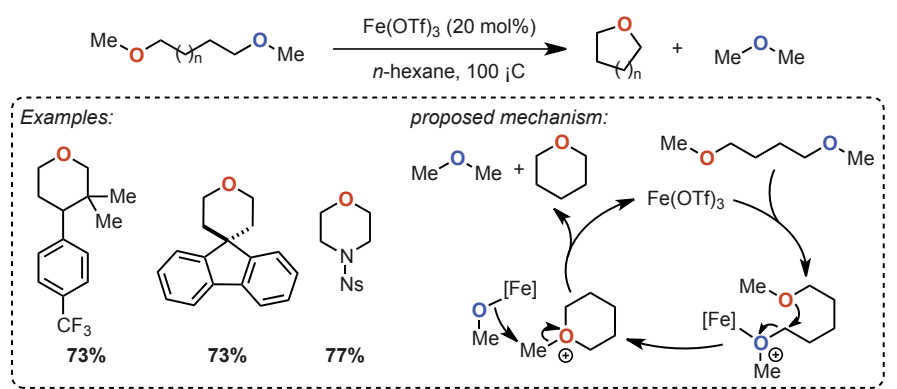

Scheme 7. Palladium-catalyzed C-P/C-P metathesis and iron-catalyzed C-O/C-O metathesis.

Reversible catalysis (shuttle reactions and metathesis) will also be the focus of more investigations in our group. Besides the obvious goal of applying this principle to novel reactions, particularly the more challenging ones involving the reversible cleavage of strong bonds $(\mathrm{C}-\mathrm{O}, \mathrm{C}-\mathrm{N}$, and $\mathrm{C}-\mathrm{F})$, we are also interested to harness the power of reversible reactions in a plethora of interdisciplinary applications. Most notably, such reactions can potentially find applications in the controlled synthesis of supramolecular entities and well-defined materials, as well as providing means to recycle end-of-life commodity chemicals. We also foresee the potential to combine multiple reversible reactions in a single reaction vessel to develop complex chemical networks akin to metabolic processes.

\section{Acknowledgements}

The Max-Planck Society, the Max-Planck-Institut für Kohlenforschung, ETH Zurich, the ERC (ShuttleCat, Project ID: 757608) and the Swiss National Science Foundation (SNSF 184658) are greatly acknowledged for continuous support of our research programs. P. B. thanks the Fonds der Chemischen Industrie for a scholarship. B. M. wants to thank all the current and former members of the Morandi group for their contribution to the research topics highlighted in this account.

Received: July 1, 2020

[1] F. Wöhler, Ann Phys. Chem. 1828, 12, 253.

[2] E. Arceo, P. Marsden, R. G. Bergman, J. A. Ellman, Chem. Commun. 2009, 3357

[3] P. Dauban, B. Darses, A. G. Jarvis, 'Addition Reactions with Formation of Carbon-Nitrogen Bonds', in 'Comprehensive Organic Synthesis', Eds.: G. Molander, P. Knochel, Elsevier, Oxford, 2014.
[4] For selected recent examples for the synthesis of unprotected amines from unsaturated compounds, see: a) L. Legnani, B. N. Bhawal, B. Morandi, Synthesis 2017, 49, 776; b) Q.-Q. Cheng, Z. Zhou, H. Jiang, J. H. Siitonen, D. H. Ess, X. Zhang, L. Kürti, Nat. Catal. 2020, 3, 386; c) S. Guo, J. C. Yang, S. L. Buchwald, J. Am. Chem. Soc. 2018, 140, 15976; d) Z. Zhou, Q.-Q. Cheng, L. Kürti, J. Am. Chem. Soc. 2019, 141, 2242; e) Z. Ma, Z. Zhou, L. Kürti, Angew. Chem. Int. Ed. 2017, 56, 9886; f) J. Liu, K. Wu, T. Shen, Y. Liang, M. Zou, Y. Zhu, X. Li, X. Li, N. Jiao, Chem. Eur. J. 2017 23, 563; g) J. L. Jat, M. P. Paudyal, H. Gao, Q.-L. Xu, M. Yousufuddin, D. Devarajan, D. H. Ess, L. Kürti, J. R. Falck, Science 2014, 343, 61; h) D. C. Miller, J. M. Ganley, A. J. Musacchio, T. C. Sherwood, W. R. Ewing, R. R. Knowles, J. Am. Chem. Soc. 2019, 141, 16590; i) S. Govaerts, L. Angelini, C. Hampton, L. Malet-Sanz, A. Ruffoni, D. Leonori, Angew. Chem. Int. Ed. 2020, 10.1002/anie.202005652.

[5] M. A. Andersson, R. Epple, V. V. Fokin, K. B. Sharpless, Angew. Chem. Int. Ed. 2002, 41, 472 .

[6] L. Legnani, B. Morandi, Angew. Chem. Int. Ed. 2016, 55, 2248.

[7] L. Legnani, G. Prina-Cerai, T. Delcaillau, S. Willems, B. Morandi, Science 2018, 362, 434.

[8] S. Makai, E. Falk, B. Morandi, ChemRxiv. Preprint. 2020, https://doi. org/10.26434/chemrxiv.12063411.v1.

[9] L. Legnani, G. Prina Cerai, B. Morandi, ACS Catal. 2016, 6, 8162.

[10] For further recent examples on the synthesis of primary anilines through C-H amination, see: a) E. M. D'Amato, J. Börgel, T. Ritter, Chem. Sci. 2019, 10, 2424; b) H. Kim, J. Heo, J. Kim, M.-H. Baik, S. Chang, J. Am. Chem. Soc. 2018, 140, 15976; c) Y. Y. See, M. S. Sanford, Org. Lett. 2020, 22, 2931; d) R. R. Anugu, S. Munnuri, J. R. Falck, J. Am. Chem. Soc. 2020, 142, 5266 e) M. P. Paudyal, A. M. Adebesin, S. C. Burt, D. H. Ess, Z. Ma, L. Kürti, J. R. Falck, Science 2016, 353, 1144.

[11] E. Falk, S. Makai, T. Delcaillau, L. Gürtler, B. Morandi, Angew. Chem. Int. Ed. 2020, doi: 10.1002/anie.202008247.

[12] A. Corma, S. Iborra, A. Velty, Chem. Rev. 2007, 107, 2411

[13] A. McNally, Nat. Chem. 2015, 7, 539.

[14] N. Drosos, B. Morandi, Angew. Chem. Int. Ed. 2015, 54, 8814.

[15] G.-J. Cheng, N. Drosos, B. Morandi, W. Thiel, ACS Catal. 2018, 8, 1697.

[16] N. Drosos, G.-J. Cheng, E. Ozkal, B. Cacherat, W. Thiel, B. Morandi, Angew. Chem. Int. Ed. 2017, 56, 13377.

[17] H.-S. Byun, L. He, R. Bittman, Tetrahedron 2000, 56, 7051.

[18] R. Ramirez-Contreras, B. Morandi, Org. Lett. 2016, 18, 3718

[19] R. H. Grubbs, 'Handbook of Metathesis', Wiley-VCH Verlag GmbH \& Co.: Weinheim, Germany, 2003.

[20] D. Wang, D. Astruc, Chem. Rev. 2015, 115, 6621.

[21] J. Cossy, S. Arseniyadis, C. Meyer, 'Metathesis in Natural Product Synthesis: Strategies, Substrates and Catalysts', Wiley-VCH Verlag GmbH \& Co.: Weinheim, Germany, 2010.

[22] K. Ivin, H. Mol, 'Olefin Metathesis and Metathesis Polymerization', Elsevier, Amsterdam, 1997.

[23] B. N. Bhawal, B. Morandi, Angew. Chem. Int. Ed. 2019, 58, 10074.

[24] L. Bini, C. Müller, D. Vogt, ChemCatChem 2010, 2, 590.

[25] X. Fang, P. Yu, B. Morandi, Science 2016, 351, 832.

[26] B. N. Bhawal, J. Reisenbauer, C. Ehinger, B. Morandi, J. Am. Chem. Soc 2020, 142, 10914.

[27] P. Yu, B. Morandi, Angew. Chem. Int. Ed. 2017, 56, 15693.

[28] X. Fang, B. Cacherat, B. Morandi, Nat. Chem. 2017, 9, 1105.

[29] P. Yu, A. Bismuto, B. Morandi, Angew. Chem. Int. Ed. 2020, 59, 2904.

[30] A. J. Cresswell, S. T. C. Eey, S. E. Denmark, Angew. Chem. Int. Ed. 2015, $54,15642$.

[31] X. Dong, J. L. Röckl, S. R. Waldvogel, B. Morandi, ChemRxiv. Preprint 2020, https://doi.org/10.26434/chemrxiv.12639215.v2

[32] a) Y.-H. Lee, B. Morandi, Nat. Chem. 2018, 10, 1016; b) M. De la Higuera Macias, B. A. Arndtsen, J. Am. Chem. Soc. 2018, 140, 10140.

[33] Z. Lian, B. N. Bhawal, P. Yu, B. Morandi, Science 2017, 356, 1059.

[34] T. Delcaillau, A. Bismuto, Z. Lian, B. Morandi, Angew. Chem. Int. Ed. 2020 59,2110

[35] T. Biberger, S. Makai, Z. Lian, B. Morandi, Angew. Chem. Int. Ed. 2018, 57, 6940.

\section{License and Terms}

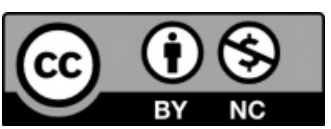

This is an Open Access article under the terms of the Creative Commons Attribution License CC BY_NC 4.0. The material may not be used for commercial purposes.

The license is subject to the CHIMIA terms and conditions: (http:// chimia.ch/component/sppagebuilder/?view=page\&id=12).

The definitive version of this article is the electronic one that can be found at https://doi.org/10.2533/chimia.2020.724 